\title{
Blood feeding tsetse flies as hosts and vectors of mammals-pre-adapted African Trypanosoma: current and expected research directions
}

\author{
Anne Geiger ${ }^{1 *}$, Imna Malele ${ }^{2}$, Adly M Abd-Alla ${ }^{3}$ and Flobert Njiokou ${ }^{4}$
}

\begin{abstract}
Research on the zoo-anthropophilic blood feeding tsetse flies' biology conducted, by different teams, in laboratory settings and at the level of the ecosystems- where also co-perpetuate African Trypanosoma- has allowed to unveil and characterize key features of tsetse flies' bacterial symbionts on which rely both (a) the perpetuation of the tsetse fly populations and (b) the completion of the developmental program of the African Trypanosoma. Transcriptomic analyses have already provided much information on tsetse fly genes as well as on genes of the fly symbiotic partners Sodalis glossinidius and Wigglesworthia, which account for the successful onset or not of the African Trypanosoma developmental program. In parallel, identification of the non- symbiotic bacterial communities hosted in the tsetse fly gut has recently been initiated: are briefly introduced those bacteria genera and species common to tsetse flies collected from distinct ecosystems, that could be further studied as potential biologicals preventing the onset of the African Trypanosoma developmental program. Finally, future work will need to concentrate on how to render tsetse flies refractory, and the best means to disseminate them in the field in order to establish an overall refractory fly population.
\end{abstract}

Keywords: Vector control, Tsetse flies, Bacteriome, Trypanosomes

\section{Background}

Human African trypanosomiasis (HAT) and animal African trypanosomiasis (AAT or nagana) are caused by flagellate protozoa belonging to the genus Trypanosoma. The parasites are transmitted to the vertebrate host (most typically a mammal) by a hematophagous insect, the tsetse fly, in which the parasites complete part of their life cycle (Fig. 1). In terms of mortality, HAT is ranked ninth out of 25 human infectious and parasitic diseases in Africa. Despite its severity, HAT is one of the most neglected tropical diseases [1].

Two subspecies, Trypanosoma brucei gambiense and Trypanosoma brucei rhodesiense, are respectively transmitted by the tsetse flies Glossina palpalis and Glossina morsitans and both are pathogenic to humans. T. b. rhodesiense causes the acute form of sleeping sickness in East

\footnotetext{
* Correspondence: anne.geiger@ird.fr

${ }^{1}$ INTERTRYP, Institut de Recherche pour le Développement, University of Montpellier, Montpellier, France

Full list of author information is available at the end of the article
}

Africa, which can lead to patient death within a few weeks if left untreated, whereas T. b. gambiense is responsible in Central and West Africa for the chronic form of the disease that develops gradually over several months to several years. The serious nature of this disease has led to it being targeted for elimination by both the WHO and the Pan-African Tsetse and Trypanosomiasis Eradication Campaign (PATTEC), and subsequently by the London Declaration on Neglected Tropical Diseases [2-5].

Recently, the number of newly diagnosed cases has begun to decrease, a situation that occurred in the 1960s that preceded the last heavy outbreak in the 1990s. Thus, despite this decrease in the detection of new cases, the severity of the situation requires continuing research to improve our current approaches (both in terms of treatment and diagnosis), as well as the development of novel control strategies that can be alternative or complementary to currently used anti-vector methods (such as mobile and stationary traps, release of sterile males). Indeed, reducing fly populations or their vector competence (i.e. rendering the fly refractory to 


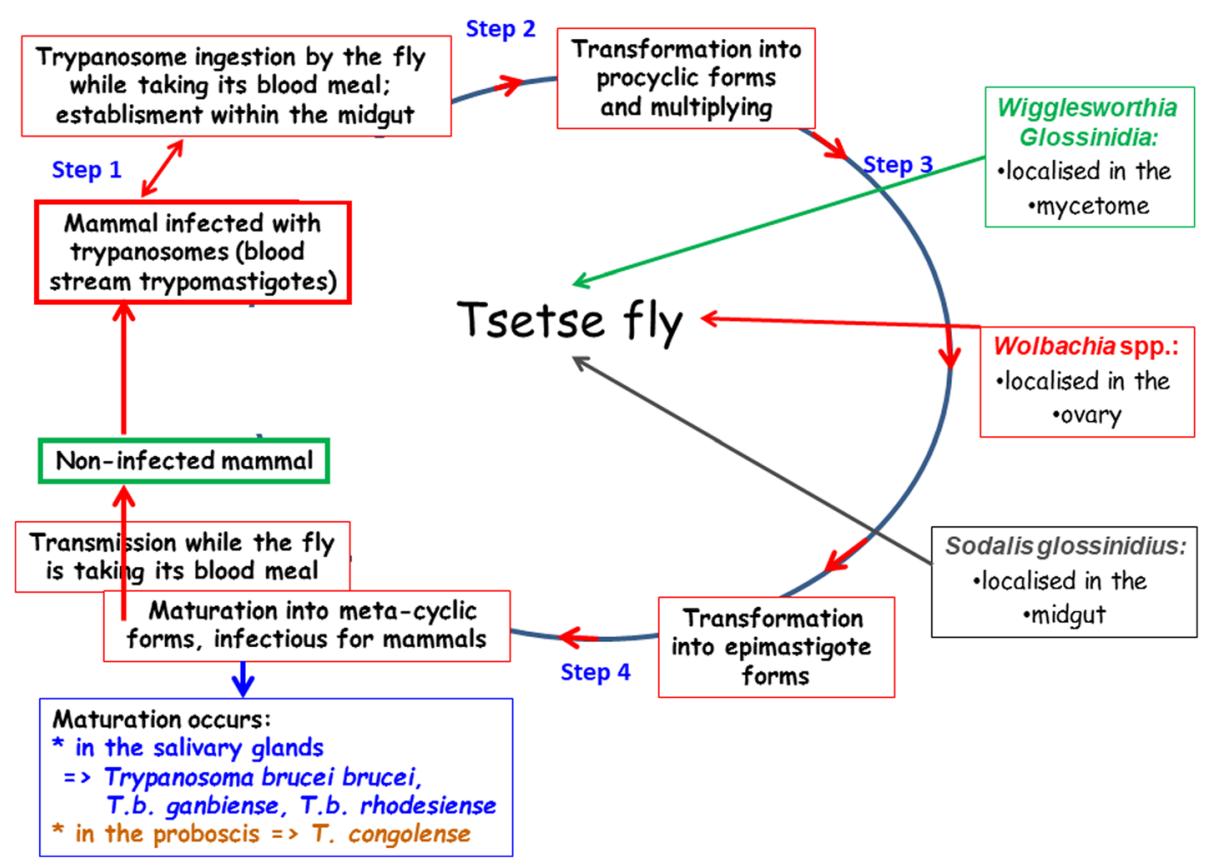

Fig. 1 Tsetse fly showing localization of its three symbionts and the trypanosome life cycle into the fly

trypanosome infection) or even the delivery of in situ trypanocidal compounds could reduce or suspend parasite dissemination, and consequently, the spread of the disease.

The successful completion of this objective requires identifying potential targets, with the caveat that some targets that are experimentally promising under laboratory conditions may not be applicable in the field operations. Included among these possible targets are the microorganisms that comprise the tsetse fly microbiome, since they likely interact with each other as well as with their host and the trypanosome and thus may contribute, positively or negatively, to tsetse infection. Furthermore, this approach will require the characterization of the mechanisms (particularly molecular) involved in these interactions, which will also necessitate determining how to stimulate or repress these mechanisms (depending on the objective). Finally, this will require testing and selecting protocols that can result in an effective, efficient, and environmentally safe field application. Here, we review these different elements (summarized in Fig. 2), some of which are already the subject of active research projects.

\section{Blood-feeding tsetse fly symbiotic and non- symbiotic bacteria communities and their potential use as biologicals to prevent the onset of African Trypanosoma developmental program} A brief overview about the early and complex tissue milieu where is initiated or not the African Trypanosoma developmental program

The unfolding or not of the pool blood feeding African Trypanosoma developmental program in the pool blood- feeding tsetse flies start when they sample their blood meal from mammals hosting tsetse fly preadapted developmental stage population: the latter populations - either Trypanosoma brucei gambiense or Trypanosoma brucei rhodensiense - or Trypanosoma congolense being delivered within the blood meal in the posterior part of the tsetse fly midgut. [6-11]. The parasites undergo proliferation and differentiation during this migration, ending in the metacyclic form, which is the only infectious form to the mammalian hosts.

The trypanosome infection rate in tsetse fly (whether natural or experimental) is low, indicating that most individuals within a fly population might be refractory to parasite infection [12-15]. In fact, the success of infection (that starts the trypanosome life cycle within its host) depends on the parasite's capacity to circumvent the tsetse immune responses. This success also depends on the host's ability to respond to the parasite's attempts to breach its first line of defense (for example by the production of trypanocidal compounds). This "game" of offensive and counter-offensive maneuvers is diverse and only ends when one of the participants takes the upper hand. Specifically, if the parasite 'wins', then it can complete its cycle and the tsetse fly performs its role as vector to transmit the trypanosome to the mammalian host. In contrast, if the fly 'wins', then this "refractory" fly will eliminate the parasite.

Though in the above $\mathbb{S}$ we got recourse to the war game metaphor, we like considering the more biologically relevant context of the unfolding or not of the African 


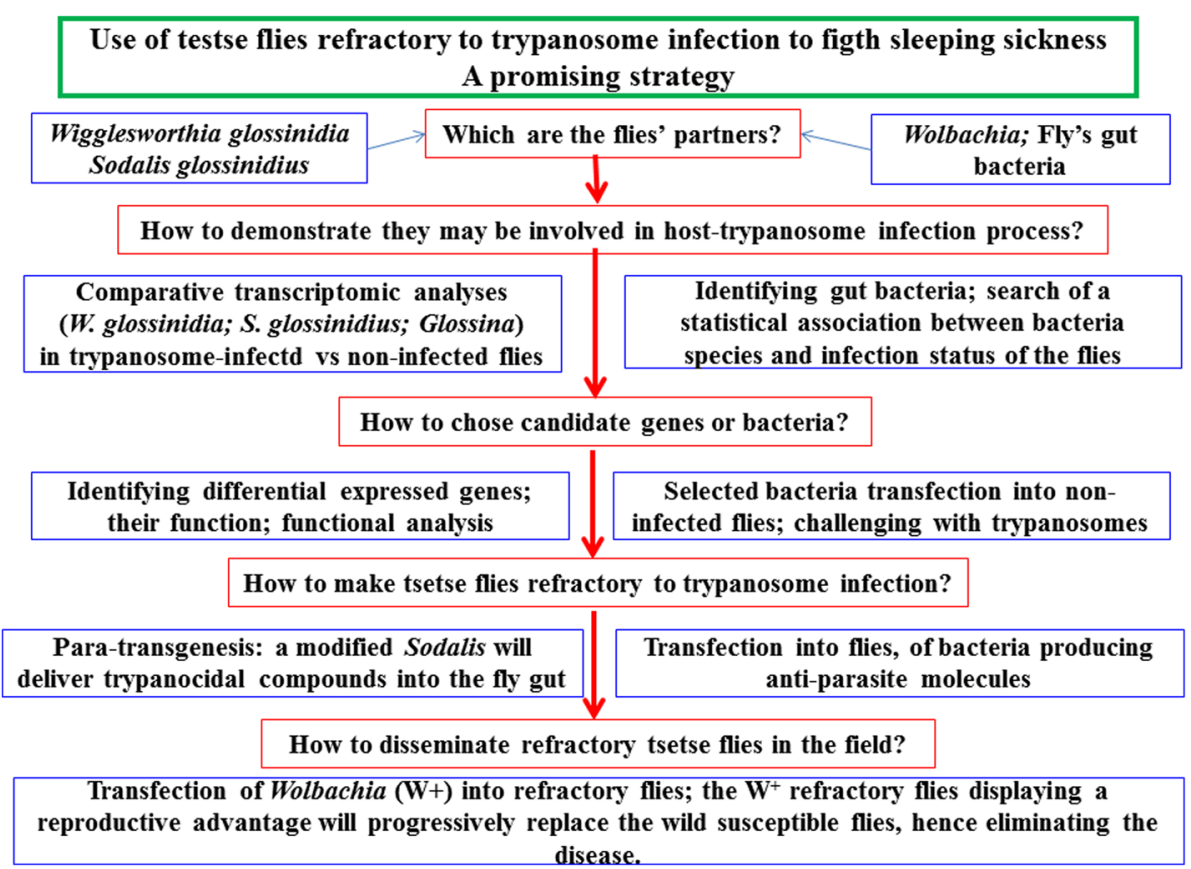

Fig. 2 Summary of the main steps involved in the "refractory fly" strategy to control sleeping sickness. Several steps (such as transcriptomic analyses, identifying of differentially expressed genes, identifying of gut bacteria from field flies) have been performed or are in progress. Similarly, technical aspects (choice of a promoter for an efficient transgene expression in Sodalis, Sodalis transfection into tsetse flies) are being solved

Trypanosoma developmental program in the tsetse fly holobiont. Such a context allows framing the Trypanosoma blood feeding -tsetse fly interactions within the context of those inter-organ communications that shape their physiology its immune and metabolic components included. The metazoan gut microbiome is increasingly recognized to play an important role in shaping and maintaining the health of the studied metazoans. Among the model fly with which to further feed this theme, Drosophila is offering potential to increase our understanding of how the microbiome influences tsetse flies' traits such as the immune system sensors and the metabolism networks [16-19].

A number of fly defense mechanisms have been reported that are involved in fly resistance to trypanosome infection, including innate immune responses, the production of reactive oxygen and antimicrobial compounds, the involvement of lectins, and Immune Deficiency (Imd) pathway-regulated gene expression [20-26]. Conversely, "silencing" Glutamic Acid - Proline (EP) protein will favor the trypanosome infection [20]. In fact, it was suggested the (EP) protein to act as a lectin [27] agglutinating trypanosomes. In addition, it was demonstrated that tsetse EP protein was strongly up regulated following stimulation with Escherichia coli [28] providing evidence that it is part of the immune response of the flies. However, the precise mechanism of its action on vector refractoriness needs still to be elucidated. Another fly defense is the proventriculus, an active insect immune tissue [29] that challenges the trypanosome transmission to the salivary glands (few trypanosomes can pass through it), where they undergo differentiation to the metacyclic form. Along Hao et al. [22] microbial challenging of tsetse flies stimulates the proventriculus production of not only antimicrobial peptides (attacin and defensin) but also of reactive nitrogen and oxygen intermediates that in turn may be responsible for the activation of other immune genes involved in refractoriness. Examples cited above are the fly's "weapons", but little is known about how trypanosomes circumvent the immune response during the various stages of their development in tsetse tissues and organs; thus trypanosome's "weapons" still need to be clarified.

The blood-feeding tsetse fly symbiotic and non-symbiotic bacteria communities hosted within their gut: Their impact on the onset or not of the developmental program of African Trypanosoma

For a long time, only three bacteria species were identified as inhabitants of the tsetse fly gut: the obligate symbiont Wigglesworthia glossinidia (Enterobacteriaceae), and the facultative symbionts Sodalis glossinidius (Enterobacteriaceae) and Wolbachia (Rickettsiaceae). However, recently, more systematic investigations using either culture-dependent or molecular (culture-independent) approaches have been performed to demonstrate that the fly microbiome is far more rich and 
diverse, as it will be shown below. Although the interactions between the microbial symbionts and their insect host (including their possible interference with the host's susceptibility to trypanosome infection) have been actively studied in the past, these types of investigations are almost absent today regarding recently identified bacteria from the intestinal flora.

\section{Wigglesworthia glossinidia}

All Glossina flies harbor the obligatory symbiont Wigglesworthia [30, 31]. This symbiont is localized intracellularly in specialized host cells (bacteriocytes) in the anterior midgut forming the bacteriome [32]. An additional extracellular population of Wigglesworthia is also found in milk glands of the female fly, allowing maternally transmission of the bacterium to the fly's offspring [33]. In contrast to the tsetse fly, the enzymes of several vitamin biosynthetic pathways are encoded by $W$. glossinidia, thus, the main role of this symbiont within the fly is to produce and provide its host with vitamins that are normally absent from the tsetse blood diet [34-37]. Wigglesworthia (Wgm) also has an immunomodulatory effect in tsetse. Specifically, Glossina morsitans morsitans flies devoid of the symbiont were shown to be immunodepressed as compared to wild flies $[36,38,39]$. Since the symbiont is involved in fly immunity, it also participates in its susceptibility to trypanosome infection. The host's peptidoglycan recognition protein LB (PGRP-LB), a catalytic member of the PRGP family [40], which cleaves bacterial peptidoglycan into non-immunogenic fragments thus preventing stimulation of the fly's Imd pathway which otherwise would have a deleterious effect on maintaining the fly's symbiosis with Wgm $[38,41]$. It must be noted that PGRP-LB expression is Wigglesworthia-dependant. Since PGRP-LB also has trypanocidal activity, increased concentrations of this protein could result in the increased refractoriness of tsetse flies to trypanosome infection, in non-teneral tsetse flies.

\section{Sodalis glossinidius}

S. glossinidius is a commensal symbiont found in all insectary-raised tsetse flies [42-44]. This bacterium is vertically transmitted through the milk gland secretions of the female tsetse to its offspring. Sodalis displays a wide tissue tropism, and is found intracellularly and extracellularly in the tsetse intestine, hemolymph, and salivary glands [33].

This symbiont is of great interest to the tsetse fly infection mechanism, since epidemiological surveys in several HAT foci indicate that Sodalis favors fly infection by trypanosomes [45]. In addition to the observation that Sodalis populations are not genetically homogeneous, it has been reported that: the genetic structure of these populations depends on the tsetse species host [43]; the population structure could differ between HAT foci [46]; and finally, there is a relationship between a given symbiont genotype and the fly infection with a given trypanosome species [44]. These results demonstrate the complex genetically-based association between the symbiont and the trypanosome infection in tsetse fly, which governs (or at least modulates) the vector competence of the Glossina fly and thus the spread of sleeping sickness. This indicates that Sodalis has potential as a target for vector control.

\section{Wolbachia}

The symbiotic bacterium Wolbachia has been reported to infect approximately $40 \%$ of all arthropods [44-49]. It is localized intracellularly in the reproductive organs, and primarily transmitted vertically by the female to her offspring [31]. Wolbachia generates reproductive abnormalities in the infected host, such as cytoplasmic incompatibility (CI), feminization, and parthenogenesis; it can also intervene in host fertility, immunity, longevity, and development [50-53]. Tsetse populations host genetically distinct but closely related Wolbachia strains $[54,55]$. It is particularly interesting that Wolbachia generates strong $\mathrm{CI}$ in tsetse flies, as in other arthropods [56]. Later in this review we will present how these particular interactions that the symbiont establishes with its host can be exploited as part of a vector control strategy against sleeping sickness, or possibly against other vector-borne diseases.

\section{Microbiota bacteria, other than Sodalis, Wigglesworthia and Wolbachia Identification and diversity}

Investigations into the possible presence of bacteria other than the three symbionts described above in the tsetse fly gut have only recently begun. In fact, most work has focused on the fly microbiome composition, following previous results on the role of the symbionts in tsetse infection with trypanosomes. This raises the issue: if symbionts have a role in the infection process, are any other gut bacteria implicated as well? Geiger et al. used bacterial isolation and culture-dependent approaches to isolate and identify a novel bacteria species (Serratia glossinae) in insectary-raised Glossina palpalis gambiensis flies [57], and several bacteria species from the gut of different Glossina species that were sampled in Angola and Cameroon [58, 59]. Most of the bacterial species belonged to the Enterobacter, Enterococcus, and Acinetobacter genera, whereas others (from flies sampled in Cameroon) belonged to Providencia, Sphingobacterium, Chryseobacterium, Lactococcus, Staphylococcus, and Pseudomonas. Similar investigations were performed on Glossina fuscipes flies sampled in Kenya [60] and Uganda [61], using either culture-dependent or 
molecular approaches (i.e. deep sequencing of the V4 hypervariable region of the $16 S$ rRNA gene) to identify a large number of bacterial genera. Finally, using similar approaches, a related study is currently in progress on Glossina pallidipes flies sampled from Tanzania (Malele I, Nyingilili H, Lyaruu E, Tauzin M, Ollivier B, Fardeau M-L, Geiger A: Bacterial diversity in the gut of G. pallidipes population from a non-sleeping sickness focus in Tanzania and its implication for species' vectorial capacity, in preparation), in which bacteria from the genera Bacillus, Acinetobacter, Mesorhizobium, Paracoccus, Microbacterium, Micrococcus, Arthrobacter, Corynobacterium, Curtobacterium, Vagococcus, and Dietzia have been identified.

Could the non-symbiotic bacteria be genetically engineered to prevent the tsetse flies to act as hosts and vectors of African Trypanosoma?

As the first stage of trypanosome infection takes place in the gut of tsetse flies, the discovery of these gut bacteria and their potential use as targets for vector control clearly represents an exciting future. In fact, a number of the isolated bacteria are reported to affect insect survival and/or vector competence (such as in: Anopheles albimanus / Serratia marcescens / Plasmodium vivax; Anopheles funestus / Gram-positive bacteria / Plasmodium falciparum; Rodnius prolixus / S. marcesens / Trypanosoma cruzi) [62]. The production of anti-parasitic molecules by bacteria species similar to those present in the tsetse fly gut has been frequently reported, including cytotoxic metalloproteases produced by S. marcescens and Pseudomonas aeruginosa [63], or hemolysins secreted by Enterobacter spp., E. coli, S. marcescens, and Enterococcus spp. [64, 65]. Furthermore, Serratia spp. produces antibiotics [66], while $P$. aeruginosa produces hemagglutinins [67] and siderophores [68], and Pseudomonas fluorescens produces an anti-T. cruzi factor [69]. Finally, pigments such as prodigiosin, which is toxic to $P$. falciparum [70] and T. cruzi [62], are produced by gram-negative bacteria such as Serratia spp. and Enterobacter spp. [71]. In the mosquito-Plasmodium model, an enterobacterium, Enterobacter spp., was recently isolated in Zambia from a mosquito species resistant to infection by $P$. falciparum, and it was suggested that the anti-Plasmodium effect is due to the production of active oxygen by this bacterium [72]. Bacteria may also have indirect roles in insects. For example, Dong et al. [73] suggested that the anti-plasmodium effect mediated by bacteria may be due to the anti-bacterial immune response of the mosquito, potentially through the activation of its immune defenses. There is a great diversity of bacteria harbored by the tsetse fly gut that may produce themselves or trigger the production by their host of an equally large variety of compounds that could be harmful or beneficial to the trypanosome, to the coinhabiting gut bacteria, or even to the vector itself. While any of these compounds could be useful to combat the parasite and consequently the disease, it will be difficult to determine how to exploit them in practice.

\section{Could a combination of bacterial community members and their phageomes hosted in the gut of Glossina morsitans morsitans or Glossina palpalis gambiense concur preventing African Trypanosoma to initiate and complete their developmental program?}

As discussed above, investigations on physiological, chemical or molecular interactions have been focused on targeted events, making it difficult to obtain an overview of the complex phenomena that occur in vivo. Indeed, the current research trend is aimed at metabolomics, proteomics and genomics approaches that are more global in nature. These approaches have been made possible thanks to technological advances in: genome sequencing and annotation; the identification of both simple and complex biological molecules; and bioinformatics and biostatistics analyses.

In this respect, sequencing and annotation (although not yet complete) of the G. m. morsitans genome [74] represents a major step, along with the genome sequencing of trypanosomes [75-78] and symbionts [34, 79, 80]. Importantly, the sequencing of the complete genomes of other tsetse species will allow a comparative analysis (and perhaps identification) of the genes that control the tsetse susceptibility to infection by a particular trypanosome species (among other events).

Two other global approaches have been developed in recent years. The first approach consisted in the analysis of the transcriptomes of the tsetse fly and its partners (today they only include S. glossinidius, Wigglesworthia, and T. $b$. gambiense) in order to identify the molecular dialogue and the disruptions induced by trypanosome infection. The genes associated with this event are differentially expressed following infection, with reference to the expression of the same genes in uninfected or refractory flies. Some of these genes could be involved in controlling fly susceptibility or refractoriness to infection (i.e. fly vector competence). The second approach consisted in analyzing the trypanosome secretome to identify the excreted/secreted proteins (ESPs) released by the parasite into the tsetse fly gut and that may participate in its establishment. Finally, all of these approaches aim to decipher the molecular dialog occurring in vivo between the partners, in order to identify the molecular steps that could be considered as targets to inhibit or at least reduce fly vector competence.

\section{Major achievements of the transcriptomics approach Sodalis glossinidius}

The S. glossinidius genome comprises 2,683 genes that are distributed across one chromosome (representing 2,523 genes) and four plasmids (accounting for 91, 31, 25, and 13 genes). Analysis of S. glossinidius inhabiting 
trypanosome-infected flies revealed 176 differentially expressed genes (DEGs), which encode a large variety of proteins including type III secretion system proteins $[81,82]$. The corresponding genes are overexpressed in the symbionts of refractory tsetse flies and results in the enrichment of the KEGG pathway "bacterial secretion systems". The effective role of the type III secretion system (injectisome) has been reported in the case of $P$. aeruginosa, which secrete toxic proteins in the cytosol of target cells [83]. Moreover, an overexpression of lysis protein transcripts was recently revealed in Sodalis harbored by refractory flies, with a corresponding enrichment in the "cytolysis, lysozyme activity, catabolism of peptidoglycan, bacteriolytic enzyme" functions $[81,82]$. In this case, the degradation products of the bacterial cell wall peptidoglycan may be involved in activation of the immune system of tsetse flies. In fact, the lysis protein transcripts were encoded by a phage genome which indicates that the symbionts were initially lysogenic and that the prophage has been activated via an unknown mechanism allowing its genes to be expressed [72, 73]. This mechanism could indirectly impact the trypanosome ability to establish infection.

\section{Wigglesworthia}

The Wigglesworthia genome comprises 673 genes. Hamidou Soumana et al. [84] determined that more than 200 genes are differentially expressed when the symbiont is hosted in infected vs. non-infected tsetse flies. Identification of the biological functions (term Gene Ontology: GO) revealed an enrichment of the GO "metabolic and binding processes" at day 3 post fly feeding on an infected meal. At day 10 post fly infection, the enriched functions were those involved in "processes of development, morphogenesis and cellular networks processes". At day 3 post fly feeding, Wigglesworthia genes encoding GroEL and GroES chaperones, non-coding RNAs, proteins involved in the transport of bacterial toxins, and proteins involved in thiamine synthesis were all under-expressed. Genes encoding GroEL and GroES chaperones were nevertheless overexpressed in the symbionts from flies at day 10 post infected meal ingestion [84]. These chaperones could function as trypanosome toxins, as shown for Enterobacter aerogenes (a symbiont of the antlion), which produces a toxic chaperone that paralyzes the antlion's prey [85]. Finally, we note that from a general point of view, the expression of a given gene may vary considerably with infection time.

\section{The first insights from comparative analysis of Glossina morsitans morsitans and Glossina palpalis gambiense genomes provide promising directions}

In 2014, given the absence of any sequenced or annotated G. p. gambiensis genome, investigations have had to rely on a RNA-seq de novo assembly approach. This approach was successful in identifying DEGs in infected vs. non-infected flies on days 3 (1,373 DEGs), 10 (52 DEGs), and 20 (1,025 DEGs) post feeding [86]. DEGs were either over- or under-expressed, and they included genes encoding proteins that exhibit a large variety of activities. The most represented genes were proteases (and some protease inhibitors), as well as oxidases (i.e. laccases), lectins, hydrolases (i.e. chitinases), and anti-microbial peptides/ proteins (such as Pro3 protein, transferrin, mucin, attacin, cecropin, etc.). As discussed in Hamidou Soumana et al. [86], a number of these up- or down-regulated proteins could be involved in tsetse fly susceptibility or refractoriness to trypanosome infection. Several examples of mechanisms that could mediate these processes include the triggering of fly immune defenses, the hydrolysis of fly protective structures such as the peritrophic matrix, the oxidative detoxification of toxic molecules, or the pathogen recognition process. The fact that both chronic and acute forms of sleeping sickness exist in Africa, caused respectively by $T . b$. gambiense and $T . b$. rhodesiense and transmitted respectively by G. p. gambiensis and G. $m$. morsitans, suggests that common molecular approaches could be developed to identify common targets to fight the disease. Indeed, one recent study was conducted to determine whether the G. m. morsitans genome carries any genes that are orthologous to DEGs in G. p. gambiensis. Specifically, the RNA-seq de novo assembled sequences from G. p. gambiensis were first mapped onto the G. m. morsitans genome in order to detect G. m. morsitans genes that are orthologous to G. p. gambiensis genes, with a special focus on the DEGs. Next, corresponding genes were annotated with respect to various databases. This approach revealed that around $50 \%$ of the G. p. gambiensis DEGs have orthologous genes in the G. m. morsitans genome [87]. Most of the G. p. gambiensis DEGs from this study that were considered to be of potential interest to an anti-vector strategy had a heterologous gene in G. m. morsitans. This list includes (but is not limited to) genes that encode proteases, chitin binding proteins, factors involved in fly immunity or antimicrobial peptide production (such as Pro3 protein), transferrin, mucin, attacin, and cecropin.

\section{Investigating trypanosome transcriptomics and proteomics}

Recently, the transcriptomes of infected G. p. gambiensis flies and the T. b. gambiense that they harbor were collectively analyzed and the corresponding genes were annotated [86]. As observed elsewhere, the genes encoding proteases were the predominant group (including aminopeptidases, aquaporin, aspartyl-peptidases, calpain, metalloproteases, and serine proteases), along with genes encoding protease inhibitors (such as cysteine 
peptidase inhibitor). Interestingly, genes encoding enzymes involved in proline metabolism (such as proline oxidase and delta-1-pyrroline-5-carboxilate dehydrogenase) and the translationally controlled tumor protein (TCTP) were expressed. All of these proteins (and many others not cited here) were found to be excreted / secreted in vitro by trypanosomes when cultivated in a secretion-stimulating medium. Here, the proteases were highly predominant in the secretomes of procyclic forms of either Tbg or T. b. brucei [88], as well as in the bloodstream strains of T. b. gambiense [89].

Finally, it must be noted that TCTP was also characterized in the secretomes of diverse Tbg forms. This protein is ubiquitously distributed in eukaryotes [90] and appears to play important roles in many biological processes such as the cell cycle, apoptosis, embryo development, cell proliferation, and stress responses [91-96]. Recent study has demonstrated that TCTP also acts on bacteria isolated from the tsetse gut, and is able to modulate their growth rate in vitro [97].

\section{Evidence that phages and other viruses could regulate the tsetse fly microbiome composition and thus modulate fly vector competence}

One recent review has provided a comprehensive of how bacteriophages could regulate bacterial communities (with consequences on human health), and how the microbial ecosystem functions [98]. This review raises the question of whether what is known about humanmicrobe relationships could be extended to microbeinsect relationships (and particularly the tsetse fly). The role of phages in modulating interactions between pathogens or pests and their hosts has often been reported. This occurs, for example, in aphids harboring the optional endosymbiont Candidatus Hamiltonella defensa, which enables the host to survive when attacked by a parasitoid wasp [99]. In fact, different strains of $H$. defensa that vary in their host protective level have been characterized, which was shown to depend on the presence of symbiont strains infected by bacteriophage, Acyrthosiphon pisum secondary endosymbiont (APSE) in a lysogenic phase [100]. Aphids hosting the $H$. defensa symbiont infected with APSE are significantly more resistant to parasitoid wasps than those that host the uninfected symbiont. The host protection was shown to be due to the production of a toxin encoded by the bacteriophage genome that is directed against eukaryotes which, in this particular case, is killing wasp larvae [101-103].

Three observations about tsetse flies require further discussion: the fact that $S$. glossinidius significantly favors fly infection by trypanosomes [45]; the fact that phage virions have been identified sporadically in cultures of S. glossinidius isolated from G. m. morsitans
[104]; and the fact that in S. glossinidius from flies refractory to trypanosome infection, several genes belonging to bacteriophage genomes were highly overexpressed as compared to S. glossinidius from trypanosome infected (and thus susceptible) flies [81]. As discussed earlier in this review, this indicates that Sodalis is lysogenic and that the prophage carried by this symbiont within the refractory fly has been activated. This allows expression (using the bacterial biosynthetic machinery) of the phage genes involved in phage nucleic acid replication, capsid protein biosynthesis, and, importantly, in bacterial cell wall peptidoglycan hydrolysis, whose degradation products are effective stimulators of insect immune defenses [105]. The authors of this study concluded that the Sodalis phage could reduce tsetse fly infection by trypanosomes, in terms of stimulating tsetse immune defenses and by reducing the symbiont density in the tsetse gut. This latter effect is in line with previous observations that flies with a higher Sodalis load are more susceptible to trypanosome infection than others [105]. It should be noted that Welburn and Maudlin [106] refer to the bacterium as a "Rickettsia-like organism"; this "organism" was later identified as S. glossinidius [107]. Currently, the mechanism that induces prophage activation in vivo within the tsetse gut remains unresolved.

Besides, Salivary Gland Hypertrophy Virus (SGHVs) has been identified that infect Glossina sp. (GpSGHV). It is the type-species of the Hytrosaviridae family of insect viruses [108]. The virus replicates in the salivary glands, causes the hypertrophy of this organ and induces reproductive dysfunction $[109,110]$, thus impairing mass production of sterile male tsetse flies in the frame of the Sterile Insect Technique (SIT strategy) to control sleeping sickness. Peacock et al. [111] observed an association between salivary gland hypertrophy symptoms and trypanosome colonization of G. pallidipes salivary glands; several authors suggested viral infection to reduce the immune defenses in this organ, thus favoring its susceptibility to trypanosome infection $[109,112,113]$. In addition, Kariithi et al. [114] reported the absence of Wolbachia, in flies displaying salivary gland hypertrophy symptoms; this may also affect tsetse fly infection.

\section{Brief review of the methods /approaches for generating Glossina morsitans morsitans and Glossina palpalis gambiense populations engineered to durably replace the native tsetse flies perpetuating in distinct ecosystems}

The current state of knowledge regarding fly/microbiome/trypanosome interactions suggests that tsetse flies could be made refractory to trypanosome infection. In this section we review the most promising methods to potentially accomplish this: mutagenesis, 
paratransgenesis, and resistance induction by intestinally residing bacteria.

\section{Mutagenesis: The CRISPR/Cas9 system}

Recent years have seen the development of a novel molecular tool for genome editing known as Clustered Regularly Interspaced Short Palindromic Repeat (CRISPR). Regarding insect research, CRISPR has been especially applied to Drosophila melanogaster, Bombyx mori, and Aedes aegypti. CRISPR generates RNAs from bacterial genomes that, when associated to nucleases, will guide these enzymes to complementary sequences of foreign DNA (such as DNA from invading pathogens); when cleaved, the foreign DNA will induce deletions or insertions in the corresponding genome (for a comprehensive recent review, refer to [115]). The CRISPR approach is expected to become a powerful tool for directed mutagenesis, with useful applications in functional analysis and introducing genes of interest (such as toxins targeted at invading pathogens) into the genome of a given organism. Thus, besides its use as a tool for genetic engineering, CRISPR could be used to fight pests and pathogens, including vector-borne diseases. There are however some restrictions. For a practical application against pathogens in the field, the host that has been genetically transformed by CRISPR must be able to transmit the beneficial character to the next generation, in order to progressively replace the susceptible population by a resistant one. Thus, for sexually reproducing organisms, the mutation must target the germ cells. However, the fact that tsetse flies are viviparous (i.e. the development of the embryos take place in utero) indicates that manipulation by CRISPR is currently not feasible (at least very difficult to perform as it would need germ-line transformation in utero).

\section{Paratransgenesis}

As opposed to genetic transformation of the tsetse fly target organism, paratransgenesis makes use of a compound of interest (such as toxins, immune responsestimulating molecules, gene repressors/activators, or enzyme activity inhibitors/stimulators) that is introduced in vivo into the fly by a microorganism which after being suitably selected or engineered, will be injected into the tsetse gut. This approach was suggested by Rio et al. [116], who considered the secondary symbiont $S$. glossinidius to be well-adapted for such a "paratransgenesis" strategy. This choice is supported by five lines of evidence: the symbiont grows in the fly gut and hemolymph as do trypanosomes [42]; it can be isolated, cultivated in vitro, and genetically transformed $[42,107,117,118]$ as it was shown by De Vooght et al. [118], when introducing a functional anti-trypanosome nanobody; it can be reintroduced into the fly [119]; it is most frequently transmitted maternally to the offspring $[33,120]$, despite the recent demonstration of paternal transmission during mating [121]; and finally, due to the large-scale erosion of its genome [122, 123], Sodalis is metabolically dependent on its tsetse fly host, suggesting that no gene flow towards any other organisms will occur once the tsetse fly harboring the modified symbiont is distributed in HAT foci.

\section{Tsetse fly refractoriness induced by intestinally residing bacteria}

In insects, gut bacteria can affect interactions with a parasite. For example, the level of susceptibility in bumble bees to the Crithidia bombi parasite is determined by the specific composition of the host intestinal microflora [124]. The tsetse fly microbiome is composed of diverse bacteria that can vary greatly according to the geographic/ecological situation of the investigated HAT foci. However, no statistically significant association between microbiome composition and the environment has been demonstrated yet, possibly due to overly restrictive sampling (i.e. the number of flies and foci, a lack of environmental contrasts, etc.). Thus, a broad sampling campaign is currently being performed on the vectors of malaria (anopheles) and sleeping sickness (tsetse flies), which associates networks of scientific partners from countries endemic or not for both diseases. The aims include: identifying all bacteria hosted by either the mosquitoes or the flies; determining whether there is an association between microbiome composition, vector infection status, and the environment; and determining if there is an association with a specific bacterium, or a restricted number of bacterial species. Once any such bacteria are identified, their physiological characteristics will need to be determined, especially regarding their potential involvement in the fly infection process (and thus vector competence) and their transmission from one fly generation to the next. Thus, the use of such natural intestinal "anti-parasitic" bacteria could be a suitable alternative to paratransgenesis.

\section{Methods to disseminate refractory tsetse flies in the field}

Once there are available tsetse flies that harbor transformed symbionts or anti-parasitic bacteria, it will be necessary to determine how to effectively and efficiently disseminate these flies in HAT foci of interest. One classical approach is that used in the so-called "sterile insect technique (SIT) strategy" relying on the release in a very large number of such engineered sterile males in the trypanosome infected foci. However, this would require the massive and periodic production and release of flies in order to overcome the natural female fly population of the target focus (and perhaps females coming from neighboring foci as well). 
One promising alternative approach suggested by Alam et al. [56] takes advantage of the induction produced by Wolbachia under specific conditions, resulting in a strong cytoplasmic incompatibility in the host. Briefly, this incompatibility occurs when a Wolbachia-negative female tsetse fly (W-) mates with a Wolbachia-positive male tsetse fly $(\mathrm{W}+)$, resulting in embryonic development failure and the absence of any progeny. In contrast, crossing a $(\mathrm{W}+)$ female tsetse to a $\left(\mathrm{W}_{+}\right)$or $\left(\mathrm{W}_{-}\right)$male results in viable, fertile progeny that are more numerous than if a (W-) female is crossed with a (W-) male. Thus, in accordance with their reproductive advantage, the dissemination in a focus of $(\mathrm{W}+)$ females that host the modified Sodalis symbiont will result in the progressive replacement of the natural population by a population of modified tsetse flies refractory to trypanosome infection. According to Alam et al. [56], a dissemination corresponding to $10 \%$ of the natural population should lead to the replacement of $90 \%$ of this population within only 2 years.

\section{Before concluding, some data highlighting the presence, in the dermis of healthy individuals, of Trypanosoma brucei gambiense transmissible to the tsetse flies}

Biologically relevant experimental studies conducted in laboratory mice to which were delivered Trypanosoma brucei brucei recently allowed documenting the prolonged presence in the mouse dermis of both slender and stumpy developmental stages. Then, they conducted an extensive histological analysis of archived skin biopsies collected from human individuals without a history of HAT enrolled in the in the Democratic Republic of the Congo as part of a screening programme for river blindness. Out of several thousands skin biopsies $0.5 \%$ trypanosomes were detected in the extracellular matrix of the vascularized dermis, namely the compartment where the pool blood feeding tsetse flies are known to sample their blood meal from the blood pool they generate: the motile stumpy developmental stage population is expected to be sampled from this blood pool [125, 126].

The occurrence of asymptomatic infections is actively monitored and documented [127]; their impact should not be underestimated. However, continued efforts are needed to refine methods to detect these infections and to evaluate the rate of transmission, to pre-adapted flies, of African Trypanosomes from the dermis of mammals from which these flies sample their blood meals.

\section{Conclusions}

Despite a decrease in the number of diagnosed cases, sleeping sickness continues to inflict a heavy burden on the people of Africa living in or near trypanosomeinfested areas. For this reason, new research projects are continually and actively developed to control and possibly eradicate the disease, with financial support from a variety of agencies (including the WHO, PATTEC, IAEA, various African, European and American Research Institutes, the Departments of Health in afflicted African countries, and the Bill and Melinda Gates Foundation).

This review has focused on diverse approaches useful for identifying molecular or bacterial targets that may make it possible to render the tsetse fly refractory to trypanosome infection, in order to block parasite transmission to humans and animals. Transcriptomic analyses of the tsetse fly and its Sodalis glossinidius and Wigglesworthia partners have provided a wealth of information on the genes that are associated with tsetse susceptibility or refractoriness to trypanosome infection. However, this only represents part of the final goal. Indeed, it is still necessary to verify the results recorded under artificial infection conditions with flies sampled in the field. Most importantly, targets must be selected and tested for their relevance and effectiveness through functional analyses. Similarly, after identifying the bacteria that compose the fly microbiome, suitable candidates must be selected and tested for their possible effectiveness on fly vector competence. Finally, extensive continuing research is necessary in order to obtain tsetse flies that are refractory to infection, so that their distribution in the field can render the overall tsetse population refractory. In addition, this method will present the advantage to preserve the pre-existent environmental biodiversity as the objective will consist in the replacement of the susceptible (or possibly susceptible) individuals by their refractory counterparts, not in the elimination of the fly populations. The control (and possible eradication) of HAT is the final goal, in order to protect people from the devastation of sleeping sickness. However, achieving this objective will require the continuing deployment of a multitude of approaches. In this spirit, it should also be kept in mind that the results obtained from sleeping sickness investigations could be used to help decipher the mechanisms of other vector-borne diseases.

\begin{abstract}
Abbreviations
AAT: animal African trypanosomiasis; APSE: Acyrthosiphon pisum secondary endosymbiont; CRISPR: Clustered Regularly Interspaced Short Palindromic Repeat; DEGs: differentially expressed genes; EP protein: Glutamic Acid Proline protein; ESPs: excreted/secreted proteins; GO: Gene Ontology; GpSGHV: Glossina sp. Salivary Gland Hypertrophy Virus; HAT: human African trypanosomiasis; Imd: Immune Deficiency; PATTEC: Pan-African Tsetse and Trypanosomiasis Eradication Campaign; PGRP-LB: peptidoglycan recognition protein LB; SGHVs: Salivary Gland Hypertrophy Virus; SIT: Sterile Insect Technique; TCTP: translationally controlled tumor protein
\end{abstract}

\section{Funding}

The authors thank the "Institut de Recherche pour le Développement", the International Atomic Energy Agency (IAEA) and "France Génomique" for their support.

Publication charges come from IAEA. 


\section{About this supplement}

This article has been published as part of BMC Microbiology Volume 18 Supplement 1, 2018: Enhancing Vector Refractoriness to Trypanosome Infection. The full contents of the supplement are available online at https://bmcmicrobiol.biomedcentral.com/articles/supplements/volume-18supplement-1.

\section{Authors' contributions}

AG, IM, AMA, FN wrote the initial manuscript, revised it and edited the manuscript. All the authors' have read and approved the final version of the manuscript.

\section{Ethics approval and consent to participate}

Not applicable.

\section{Consent for publication}

Not applicable.

\section{Competing interests}

The authors report no conflict of interest with respect to funding or any other issue.

\section{Publisher's Note}

Springer Nature remains neutral with regard to jurisdictional claims in published maps and institutional affiliations.

\section{Author details}

'INTERTRYP, Institut de Recherche pour le Développement, University of Montpellier, Montpellier, France. ${ }^{2}$ Vector and Vector Borne Diseases Institute, Majani Mapana, Off Korogwe Road, Box, 1026 Tanga, Tanzania. ${ }^{3}$ Insect Pest Control Laboratory, Joint FAO/IAEA Division of Nuclear Techniques in Food and Agriculture, Vienna, Austria. ${ }^{4}$ Faculty of Science, University of Yaoundé I, P.O. Box 812, Yaoundé, Cameroon.

\section{Published: 23 November 2018}

\section{References}

1. Brun R, Blum J, Chappuis F, Burri C. Human African trypanosomiasis. Lancet. 2010;375:148-59.

2. WHO: WHO programme to eliminate sleeping sickness - Building a global alliance. WHO 2002 http://whqlibdoc.who.int/hq/2002/WHO CDS CSR EPH_2002.13.pdf. Accessed date 01 MAY 2002.

3. The Pan African Tsetse and Trypanosomiasis Eradication Campaign (PATEC) www.who.int/trypanosomiasis_african/partners/pattec/en/ accessed date 06 JAN 2018.

4. London Declaration on Neglected Tropical Diseases: http://www.who.int/ neglected diseases/London Declaration_NTDs.pdf. Accessed date 30 JAN 2012

5. Simarro PP, Jannin J, Cattand P. Eliminating human African trypanosomiasis: where do we stand and what comes next? PLoS Med. 2008;5(2):e55 https:// doi.org/10.1371/journal.pmed.0050055.

6. Van den Abbeele J, Claes Y, Bockstaele D, Ray D, Coosemans M. Trypanosoma brucei spp. development in the tsetse fly: characterization of the post-mesocyclic stages in the foregut and proboscis. Parasitology. 1999:118:469-78.

7. Rio RVM, Attardo GM, Weiss BL. Grandeur Alliances: Symbiont Metabolic Integration and Obligate Arthropod Hematophagy. Trends Parasitol. 2016; 32(9):739-49.

8. Matetovici I, Caljon G, Van Den Abbeele J. Tsetse fly tolerance to T. brucei infection: transcriptome analysis of trypanosome-associated changes in the tsetse fly salivary gland. BMC Genomics. 2016;17(1):971.

9. Tsagmo Ngoune JM, Njiokou F, Loriod B, Kame-Ngasse G, Fernandez-Nunez N, Rioualen C, van Helden J, Geiger A. Transcriptional profiling of midguts prepared from Trypanosoma/T. congolense-positive Glossina palpalis palpalis collected from two distinct Cameroonian foci: coordinated signatures of the Midguts' remodeling as T. congolense-supportive niches. Front Immunol. 2017;8:876

10. Awuoche EO, Weiss BL, Vigneron A, Mireji PO, Aksoy E, Nyambega B, Attardo GM, Wu Y, O'Neill M, Murilla G, Aksoy S. Molecular characterization of tsetse's proboscis and its response to Trypanosoma congolense infection. PLoS Negl Trop Dis. 2017;11(11):e0006057.
11. Sloan, M.A., Ligoxygakis, P. (2017) Immunology of insect vectors: midgut interactions of sandflies and tsetse with Kinetoplastid parasites as a paradigm for establishing infection. Advances Insect Physiol 2017, 52:231-248.

12. Moloo SK, Kabata JM, Sabwa CL. A study on the maturation of procyclic Trypanosoma brucei brucei in Glossina morsitans centralis and G. brevipalpis. Med Vet Entomol. 1994:8:369-74.

13. Moloo SK, Okumu IO, Kuria NM. Comparative susceptibility of Glossina longipennis and G. brevipalpis to pathogenic species of Trypanosoma. Med Vet Entomol. 1998:12:211-4

14. Moloo SK, Karia FW, Okumu IO. Membrane feeding Glossina morsitans centralis on livestock blood and its effect on the tsetse susceptibility to pathogenic trypanosome infections. Med Vet Entomol. 1999;13:110-3.

15. Peacock L, Cook S, Ferris V, Bailey M, Gibson W. The life cycle of Trypanosoma (Nannomonas) congolense in the tsetse fly. Parasit Vectors. 2012;5:109.

16. Teleman AA, Perrimon N. Open questions: completing the parts list and finding the integrating signals. BMC Biol. 2017;15(1):47.

17. Droujinine IA, Perrimon N. Interorgan communication pathways in physiology: focus on Drosophila. Annu Rev Genet. 2016;50:539-70.

18. Leulier F, MacNeil LT, Lee WJ, Rawls JF, Cani PD, Schwarzer M, Zhao L, Simpson SJ. Integrative physiology: at the crossroads of nutrition, microbiota, animal physiology, and human health. Cell Metab. 2017;25(3):522-34.

19. Douglas AE. The Drosophila model for microbiome research. Lab Animal. 2018:47:157-64

20. Haines LR, Lehane SM, Pearson TW, Lehane MJ. Tsetse EP protein protects the fly midgut from trypanosome establishment. PLoS Pathog. 2010;6: e1000793.

21. Hao Z, Kasumba I, Lehane MJ, Gibson WC, Kwon J, Aksoy S. Tsetse immune responses and trypanosome transmission: implications for the development of tsetse- based strategies to reduce trypanosomiasis. Proc Natl Acad Sci U S A. 2001:98:12648-53.

22. Hao Z, Kasumba I, Aksoy S. Proventriculus (cardia) plays a crucial role in immunity in tsetse fly (Diptera: Glossinidiae). Insect Biochem Mol Biol. 2003; 33:1155-64

23. Hu Y, Aksoy S. An antimicrobial peptide with trypanocidal activity characterized from Glossina morsitans morsitans. Insect Biochem Mol Biol. 2005:35:105-15.

24. Hu C, Aksoy S. Innate immune responses regulate trypanosome parasite infection of the tsetse fly Glossina morsitans morsitans. Mol Microbiol. 2006; 60:1194-204.

25. Nayduch D, Aksoy S. Refractoriness in tsetse flies (Diptera: Glossinidae) may be a matter of timing. J Med Entomol. 2007;44:660

26. Welburn SC, Maudlin I. Lectin signaling of maturation of T. congolense infections in tsetse. Med Vet Entomol. 1989:3:141-5.

27. Chandra M, Liniger M, Tetley L, Roditi I, Barry JD. TsetseEP, a gut protein from the tsetse Glossina morsitans, is related to a major surface glycoprotein of trypanosomes transmitted by the fly and to the products of a Drosophila gene family. Insect Biochem Mol Biol. 2004:34:1163-73.

28. Haines LR, Jackson AM, Lehane MJ, Thomas JM, Yamaguchi AY, Haddow JD, Pearson TW. Increased expression of unusual EP repeat-containing proteins in the midgut of the tsetse fly (Glossina) after bacterial challenge. Insect Biochem Mol Biol. 2005:35:413-23.

29. Aksoy S, Gibson WC, Lehane MJ. Interactions between tsetse and trypanosomes with implications for the control of trypanosomiasis. Adv Parasitol. 2003;53:1-83.

30. Aksoy S, Pourhosseini AA, Chow A. Mycetome endosymbionts of tsetse flies constitute a distinct lineage related to Enterobacteriaceae. Insect Mol Biol. 1995:4:15-22.

31. Chen X, Li S, Aksoy S. Concordant evolution of a symbiont with its host insect species: molecular phylogeny of genus Glossina and its bacteriome-associated endosymbiont, Wigglesworthia glossinidia. J Mol Evol. 1999;48:49-58.

32. Snyder AK, Rio RV. Interwoven biology of the tsetse holobiont. J Bacteriol. 2013:195:4322-30

33. Balmand S, Lohs C, Aksoy S, Heddi A. Tissue distribution and transmission routes for the tsetse fly endosymbionts. J Invertebr Pathol. 2013;(112 Suppl): S116-22.

34. Akman L, Yamashita A, Watanabe H, Oshima K, Shiba T, Hattori M, Aksoy S. Genome sequence of the endocellular obligate symbiont of tsetse flies, Wigglesworthia glossinidia. Nat Genet. 2002:32:402-7.

35. Nogge G. Significance of symbionts for the maintenance of an optional nutritional state for successful reproduction in hematophagous arthropods. Parasitology. 1982;82:299-304. 
36. Pais R, Lohs C, Wu YN, Wang JW, Aksoy S. The obligate mutualist Wigglesworthia glossinidia influences reproduction, digestion, and immunity processes of its host, the tsetse fly. ApplEnvironMicrobiol. 2008;74:5965-74.

37. Rio RVM, Symula RE, Wang J, Lohs C, Wu YN, Snyder AK, et al. Insight into the transmission biology and species-specific functional capabilities of tsetse (Diptera: Glossinidae) obligate symbiont Wigglesworthia. mBio. 2012; 3:e00240-11.

38. Wang J, Wu Y, Yang G, Aksoy S. Interactions between mutualist Wigglesworthia and tsetse peptidoglycan recognition protein (PGRP-LB) influence trypanosome transmission. Proc Natl Acad Sci U S A. 2009;106:12133-8.

39. Weiss BL, Wang J, Aksoy S. Tsetse immune system maturation requires the presence of obligate symbionts in larvae. PLoS Biol. 2011;9:e1000619.

40. Zaïdman-Rémy A, Hervé M, Poidevin M, Pili-Floury S, Kim MS, Blanot D, Oh $\mathrm{BH}$, Ueda R, Mengin-Lecreulx D, Lemaitre B. The Drosophila amidase PGRPLB modulates the immune response to bacterial infection. Immunity. 2006; 24:463-73.

41. Wang J, Aksoy S. PGRP-LB is a maternally transmitted immune milk protein that influences symbiosis and parasitism in tsetse's offspring. Proc Natl Acad Sci U S A. 2012;109:10552-7.

42. Cheng Q, Aksoy S. Tissue tropism, transmission and expression of foreign genes in vivo in midgut symbionts of tsetse flies. Insect Mol Biol. 1999:8:125-32.

43. Geiger A, Cuny G, Frutos R. Two Tsetse fly species, Glossina palpalis gambiensis and Glossina morsitans morsitans, carry genetically distinct populations of the secondary symbiont Sodalis glossinidius. Appl Environ Microbiol. 2005;71:8941-3.

44. Geiger A, Ravel S, Mateille T, Janelle J, Patrel D, Cuny G, Frutos R. Vector competence of Glossina palpalis gambiensis for Trypanosoma brucei s.l. and genetic diversity of the symbiont Sodalis glossinidius. Mol Biol Evol. 2007;24:102-9.

45. Farikou O, Njiokou F, Mbida Mbida JA, Njitchouang GR, Djeunga HN, Asonganyi T, Simarro PP, Cuny G, Geiger A. Tripartite interactions between tsetse flies, Sodalis glossinidius and trypanosomes--an epidemiological approach in two historical human African trypanosomiasis foci in Cameroon. Infect Genet Evol. 2010;10:115-21.

46. Farikou O, Thevenon S, Njiokou F, Allal F, Cuny G, Geiger A. Genetic diversity and population structure of the secondary symbiont of tsetse flies, Sodalis glossinidius, in sleeping sickness foci in Cameroon. PLoS Negl Trop Dis. 2011:5:e1281.

47. Hilgenboecker $K$, Hammerstein $P$, Schlattmann $P$, Telschow A, Werren JH. How many species are infected with Wolbachia?--a statistical analysis of current data. FEMS Microbiol Lett. 2008;281:215-20.

48. O'Neill SL, Gooding RH, Aksoy S. Phylogenetically distant symbiotic microorganisms reside in Glossina midgut and ovary tissues. Med Vet Entomol. 1993;7:377-83.

49. Werren JH, Baldo L, Clark ME. Wolbachia: master manipulators of invertebrate biology. Nat Rev Microbiol. 2008;6:741-51.

50. Glaser RL, Meola MA. The native Wolbachia endosymbionts of Drosophila melanogaster and Culex quinquefasciatus increase host resistance to West Nile virus infection. PLoS One. 2010;5:e11977.

51. Kambris Z, Cook PE, Phuc HK, Sinkins SP. Immune activation by lifeshortening Wolbachia and reduced filarial competence in mosquitoes. Science. 2009;326:134-6.

52. Kambris Z, Blagborough AM, Pinto SB, Blagrove MS, Godfray HC, Sinden RE, Sinkins SP. Wolbachia stimulates immune gene expression and inhibits plasmodium development in Anopheles gambiae. PLoS Pathog. 2010;6:e1001143.

53. Moreira LA, Iturbe-Ormaetxe I, Jeffery JA, Lu G, Pyke AT, Hedges LM, Rocha BC, Hall-Mendelin S, Day A, Riegler M, Hugo LE, Johnson KN, Kay BH, McGraw EA, van den Hurk AF, Ryan PA, O'Neill SL. A Wolbachia symbiont in Aedes aegypti limits infection with dengue, Chikungunya, and Plasmodium. Cell. 2009;139:1268-78.

54. Cheng Q, Ruel TD, Zhou W, Moloo SK, Majiwa P, O'Neill SL, et al. Tissue distribution and prevalence of Wolbachia infections in tsetse flies, Glossina spp. Med Vet Entomol. 2000;14:44-50.

55. Doudoumis V, Tsiamis G, Wamwiri F, Brelsfoard C, Alam U, Aksoy E, Dalaperas S, Abd-Alla A, Ouma J, Takac P, Aksoy S, Bourtzis K. Detection and characterization of Wolbachia infections in laboratory and natural populations of different species of tsetse flies (genus Glossina). BMC Microbiol. 2012;12(Suppl 1):S3.

56. Alam U, Medlock J, Brelsfoard C, Pais R, Lohs C, Balmand S, et al. Wolbachia symbiont infections induce strong cytoplasmic incompatibility in the tsetse fly Glossina morsitans. PLoS Pathog. 2011;7:e1002415.
57. Geiger A, Fardeau ML, Falsen E, Ollivier B, Cuny G. Serratia glossinae sp. nov. isolated from the midgut of the tsetse fly Glossina palpalis gambiensis. Int J Syst Evol Microbiol. 2010;60:1261-5.

58. Geiger A, Fardeau ML, Grebaut P, Vatunga G, Josénando T, Herder S, et al. First isolation of Enterobacter, enterococcus, and Acinetobacter spp. as inhabitants of the tsetse fly (Glossina palpalis palpalis) midgut. Infect Genet Evol. 2009;9:1364-70.

59. Geiger A, Fardeau ML, Njiokou F, Joseph M, Asonganyi T, Ollivier B, et al. Bacterial diversity associated with populations of Glossina spp. from Cameroon and distribution within the campo sleeping sickness focus. Microb Ecol. 2011;62:632-43.

60. Lindh JM, Lehane MJ. The tsetse fly Glossina fuscipes fuscipes (Diptera: Glossina) harbours a surprising diversity of bacteria other than symbionts. Antonie Van Leeuwenhoek. 2011;99:711-20.

61. Aksoy E, Telleria EL, Echodu R, Wu Y, Okedi LM, Weiss BL, Aksoy S, Caccone A. Analysis of multiple tsetse fly populations in Uganda reveals limited diversity and species-specific gut microbiota. Appl Environ Microbiol. 2014; 80:4301-12

62. Azambuja P, Garcia ES. Ratcliffe NA:qut microbiota and parasite transmission by insect vectors. Trends Parasitol. 2005;21:568-72.

63. Maeda H, Morihara K. Serralysin and related bacterial proteinases. Methods Enzymol. 1995;248:395-413.

64. Hertle R, Hilger M, Weingardt-Kocher S, Walev I. Cytotoxic action of Serratia marcescens hemolysin on human epithelial cells. Infect Immun. 1999;67:817-25.

65. Coburn PS, Gilmore MS. The Enterococcus faecalis cytolysin: a novel toxin active against eukaryotic and prokaryotic cells. Cell Microbiol. 2003;5:661-9.

66. Thomson NR, Crow MA, McGowan SJ, Cox A, Salmond GPC. Biosynthesis of carbapenem antibiotic and prodigiosin pigment in Serratia is under quorum sensing control. MolMicrobiol. 2000;36:539-56.

67. Gilboa-Garber N. Purification and properties of hemagglutinin from Pseudomonas aeruginosa and its reaction with human blood cells. Biochim Biophys Acta. 1972:273(1):165-73.

68. Schalk IJ, Abdallah MA, Pattus F. Recycling of pyoverdinon the FpvA receptor after ferric pyoverdin uptake and dissociation in Pseudomonas aeruginosa. Biochemistry. 2002;41:1663-71.

69. Mercado TI, Colon-Whitt A. Lysis of Trypanosoma cruzi by Pseudomonas fluorescens. AntimicrobAgents Chemother. 1982;22:1051-7.

70. Lazaro JE, Nitcheu J, Predicala RZ, Mangalindan GC, Nesslany F, Marzin D, et al. Heptyl prodigiosin, a bacterial metabolite is antimalarial in vivo and non-mutagenic in vitro. J NatToxins. 2002;11:367-77.

71. Moss M. Bacterial pigments. Microbiologist. 2002;3:10-2.

72. Cirimotich CM, Dong Y, Clayton AM, Sandiford SL, Souza-Neto JA. Mulenga,M,et al: Naturalmicrobe-mediated refractoriness to Plasmodium infection in Anopheles gambiae. Science. 2011;332:855-8.

73. Dong Y, Manfredini F, Dimopoulos G. Implication of the mosquito midgut microbiota in the defense against malaria parasites. PLoS Pathog. 2009;5: e1000423.

74. International Glossina Genome Initiative. Genome sequence of the tsetse fly (Glossina morsitans): vector of African trypanosomiasis. Science. 2014;344:380-6.

75. El-Sayed NM, Myler PJ, Blandin G, Berriman M, Crabtree J, Aggarwal G, Caler E, Renauld H, Worthey EA, Hertz-Fowler C, Ghedin E, Peacock C, Bartholomeu DC, Haas BJ, Tran AN, Wortman JR, Alsmark UC, Angiuoli S, Anupama A, Badger J, Bringaud F, Cadag E, Carlton JM, Cerqueira GC, Creasy T, Delcher AL, Djikeng A, Embley TM, Hauser C, Ivens AC, Kummerfeld SK, Pereira-Leal JB, Nilsson D, Peterson J, Salzberg SL, Shallom J, Silva JC, Sundaram J, Westenberger S, White O, Melville SE, Donelson JE, Andersson B, Stuart KD, Hall N. Comparative genomics of trypanosomatid parasitic protozoa. Science. 2005;309:404-9.

76. Jackson AP, Sanders M, Berry A, McQuillan J, Aslett MA, Quail MA, Chukualim B, Capewell P, MacLeod A, Melville SE, Gibson W, Barry JD, Berriman M, Hertz-Fowler C. The genome sequence of Trypanosoma brucei gambiense, causative agent of chronic human african trypanosomiasis. PLoS Negl Trop Dis. 2010:4:e658.

77. Kolev NG, Franklin JB, Carmi S, Shi H, Michaeli S, Tschudi C. The transcriptome of the human pathogen Trypanosoma brucei at singlenucleotide resolution. PLoS Pathog. 2010;6:e1001090.

78. Sistrom M, Evans B, Benoit J, Balmer O, Aksoy S, Caccone A. De Novo Genome Assembly Shows Genome Wide Similarity between Trypanosoma brucei brucei and Trypanosoma brucei rhodesiense. PLoS One. 2016:11: e0147660. 
79. Darby AC, Lagnel J, Matthew CZ, Bourtzis K, Maudlin I, Welburn SC. Extrachromosomal DNA of the symbiont Sodalis glossinidius. J Bacteriol. 2005; 187:5003-7

80. Toh H, Weiss BL, Perkin SA, Yamashita A, Oshima K, Hattori M, Aksoy S. Massive genome erosion and functional adaptations provide insights into the symbiotic lifestyle of Sodalis glossinidius in the tsetse host. Genome Res. 2006;16:149-56.

81. Hamidou Soumana I, Loriod B, Ravel S, Tchicaya B, Simo G, Rihet P, Geiger A. The transcriptional signatures of Sodalis glossinidius in the Glossina palpalis gambiensis flies negative for Trypanosoma brucei gambiense contrast with those of this symbiont in tsetse flies positive for the parasite: possible involvement of a Sodalis-hosted prophage in fly Trypanosoma refractoriness? Infect Genet Evol. 2014a;24:41-56.

82. Hamidou Soumana I, Tchicaya B, Loriod B, Rihet P, Geiger A. Identification of overexpressed genes in Sodalis glossinidius inhabiting trypanosomeinfected self-cured tsetse flies. Front Microbiol. 2014b;5:255.

83. Cornelis GR. Type III secretion: a bacterial device for close combat with cells of their eukaryotic host. Philos Trans R Soc Lond Ser B Biol Sci. 2000;355:681-93.

84. Hamidou Soumana I, Tchicaya B, Simo G, Geiger A. Comparative gene expression of Wigglesworthia inhabiting non-infected and Trypanosoma brucei gambiense-infected Glossina palpalis gambiensis flies. Front Microbiol. 2014c;5:620

85. Yoshida N, Oeda K, Watanabe E, Mikami T, Fukita Y, Nishimura K, Komai K, Matsuda K. Protein function: chaperonin turned insect toxin. Nature. 2001; 411:44. https://doi.org/10.1038/35075148.

86. Hamidou Soumana I, Klopp C, Ravel S, Nabihoudine I, Tchicaya B, Parrinello H, Abate L, Rialle S, Geiger A. RNA-seq de novo Assembly Reveals Differential Gene Expression in Glossina palpalis gambiensis Infected with Trypanosoma brucei gambiense vs. Non-Infected and Self-Cured Flies. Front Microbiol. 2015;2015(6):1259.

87. Hamidou Soumana I, Tchicaya B, Rialle S, Parrinello H, Geiger A. Comparative genomics of Glossina palpalis gambiensis and $\mathrm{G}$. morsitans morsitans to reveal gene orthologs involved in infection by Trypanosoma brucei gambiense. Frontiers Microbiol. 2017;8:540. 28421044. https://doi.org/ 10.3389/fmicb.2017.00540.

88. Atyame Nten CM, Sommerer N, Rofidal V, Hirtz C, Rossignol M, Cuny G, Peltier JB, Geiger A. Excreted/secreted proteins from trypanosome procyclic strains. J Biomed Biotechnol. 2010;2010:212817.

89. Geiger A, Hirtz C, Bécue T, Bellard E, Centeno D, Gargani D, Rossignol M, Cuny G, Peltier JB. Exocytosis and protein secretion in Trypanosoma. BMC Microbiol. 2010;10:20. https://doi.org/10.1186/1471-2180-10-20.

90. Brioudes F, Thierry AM, Chambrier P, Mollereau B, Bendahmane M. Translationally controlled tumor protein is a conserved mitotic growth integrator in animals and plants. Proc Natl Acad Sci U S A. 2010;107: 16384-9.

91. Arcuri F, Papa S, Carducci A, Romagnoli R, Liberatori S, Riparbelli MG, Sanchez JC, Tosi P, del Vecchio MT. Translationally controlled tumor protein (TCTP) in the human prostate and prostate cancer cells: expression, distribution, and calcium binding activity. Prostate. 2004:60:130-40.

92. Bommer U, Heng C, Perrin A, Dash P, Lobov S, Elia A, Clemens MJ. Roles of the translationally controlled tumour protein (TCTP) and the doublestranded RNA-dependent protein kinase, PKR, in cellular stress responses. Oncogene. 2010;29:763-73.

93. Chen W, Wang H, Tao S, Zheng Y, Wu W, Lian F, Jaramillo M, Fang D, Zhang DD. Tumor protein translationally controlled 1 is a p53 target gene that promotes cell survival. Cell Cycle. 2013;12:2321-8.

94. Roque CG, Wong HH, Lin JQ, Holt CE. Tumor protein Tctp regulates axon development in the embryonic visual system. Development. 2016; 143:1134-48.

95. Susini L, Besse S, Duflaut D, Lespagnol A, Beekman C, Fiucci G, Atkinson AR, Busso D, Poussin P, Marine JC, Martinou JC, Cavarelli J, Moras D, Amson R, Telerman A. TCTP protects from apoptotic cell death by antagonizing bax function. Cell Death Differ. 2008;15:1211-20.

96. Stierum R, Gaspari M, Dommels Y, Ouatas T, Pluk H, Jespersen S, Vogels J, Verhoeckx K, Groten J, van Ommen B. Proteome analysis reveals nove proteins associated with proliferation and differentiation of the colorectal cancer cell line Caco-2. Biochim Biophys Acta. 2003;1650:73-91.

97. Bossard G, Bartoli M, Fardeau ML, Ollivier B, Geiger A. Characterization of recombinant Trypanosoma brucei gambiense translationally controlled tumor protein (rTbgTCTP) and its interaction with Glossina midgut bacteria. Gut Microbes. 2017; in press.
98. Mirzaei MK, Maurice C.F: Ménage à trois in the human gut: interactions between host, bacteria and phages. Nat Rev Microbiol 2017, 2017 May 2. https://doi.org/10.1038/ nmicro.2017.30.

99. Degnan PH, Yu Y, Sisneros N, Wing RA, Moran NA. Hamiltonella defensa, genome evolution of protective bacterial endosymbiont from pathogenic ancestors. Proc Natl Acad Sci U S A. 2009;106:9063-8.

100. Duron O. Arsenophonus insect symbionts are commonly infected with APSE, a bacteriophage involved in protective symbiosis. FEMS Microbiol Ecol. 2014;90:184-94.

101. Degnan PH, Moran NA. Evolutionary genetics of a defensive facultative symbiont of insects: exchange of toxin-encoding bacteriophage. Mol Ecol. 2008;17:916-29.

102. Degnan PH, Moran NA. Diverse phage-encoded toxins in a protective insect endosymbiont. Appl Environ Microbiol. 2008b;74:6782-91.

103. Oliver KM, Degnan PH, Hunter MS, Moran NA. Bacteriophages encode factors required for protection in a symbiotic mutualism. Science. 2009; 325(5943):992-4. https://doi.org/10.1126/science.1174463.

104. Clark AJ, Pontes M, Jones T, Dale C. A possible heterodimeric prophagelike element in the genome of the insect endosymbiont Sodalis glossinidius. J Bacteriol. 2007;189:2949-51.

105. Mellroth P, Karlsson J, Steiner H. A scavenger function for a Drosophila peptidoglycan recognition protein. J Biol Chem. 2003;278:7059-64.

106. Welburn SC, Maudlin I. Rickettsia-like organisms, puparial temperature and susceptibility to trypanosome infection in Glossina morsitans. Parasitology. 1991;102:201-6.

107. Dale C, Maudlin I. Sodalis gen. Nov. and Sodalis glossinidius sp. nov., a microaerophilic secondary endosymbiont of the tsetse fly Glossina morsitans morsitans. Int J Syst Bacteriol. 1999;(1):267-75.

108. Lietze V-U, Abd-Alla A, Vreysen M, Geden CC, Boucias DG. Salivary gland hypertrophy viruses: a novel group of insect pathogenic viruses. Annu Rev Entomol. 2011;56:63-80.

109. Kariithi HM, Ince Al, Boeren S, Murungi EK, Meki IK, Otieno EA, et al. Comparative analysis of salivary gland proteomes of two Glossina species that exhibit differential hytrosavirus pathologies. FrontMicrobiol. 2016;7:89.

110. Kariithi HM, Meki IK, Boucias DG, Abd-Alla AM. Hytrosaviruses: current status and perspective. Curr Opin Insect Sci. 2017;22:71-8.

111. Peacock L, Ferris V, Bailey M, Gibson W. The influence of sex and fly species on the development of trypanosomes in tsetse flies. PLoS Negl Trop Dis. 2012;6:e1515.

112. Guerra L, Stoffolano JG, Gambellini G, Masci VL, Belardinelli MC, Fausto AM. Ultrastructure of the salivary glands of non-infected and infected glands in Glossina pallidipes by the salivary glands hypertrophy virus. J Invertebr Pathol. 2013;112:S53-61.

113. Orlov I, Drillien R, Spehner D, Bergoin M, Abd-Alla AMM, Klaholz BP. Structural features of the salivary gland hypertrophy virus of the tsetse fly revealed by cryo-electron microscopy and tomography. Virology. 2018:514:165-9.

114. Kariithi HM, Boucias DG, Murungi EK, Meki IK, Demirbaş-Uzel G, van Oers $\mathrm{MM}$, et al. Coevolution of hytrosaviruses and host immune responses. BMC Microbiol. 2018; in press.

115. Taning CNT, Van Eynde B, Yu N, MA S, Smagghe G. CRISPR/Cas9 in insects: applications, best practices and biosafety concerns. J Insect Physiology. 2017. https://doi.org/10.1016/j.jinsphys.2017.01.007.

116. Rio RV, Hu Y, Aksoy S. Strategies of the home-team: symbioses exploited for vector-borne disease control. Trends Microbiol. 2004;12:325-36.

117. De Vooght L, Caljon G, Stijlemans B, De Baetselier P, Coosemans M, Van den Abbeele J. Expression and extracellular release of a functional antitrypanosome Nanobody ${ }^{\oplus}$ in Sodalis glossinidius, a bacterial symbiont of the tsetse fly. Microb Cell Factories. 2012;11:23.

118. De Vooght L, Caljon G, De Ridder K, Van Den Abbeele J. Delivery of a functional anti-trypanosome Nanobody in different tsetse fly tissues via a bacterial symbiont, Sodalis glossinidius. Microb Cell Factories. 2014;13:156.

119. Weiss BL, Mouchotte R, Rio RV, Wu YN, Wu Z, Heddi A, Aksoy S: Interspecific transfer of bacterial endosymbionts between tsetse fly species: infection establishment and effect on host fitness. 2006.

120. Wang J, Weiss BL, Aksoy S. Tsetse fly microbiota: form and function. Front Cell Infect Microbiol. 2013;3:69.

121. De Vooght L, Caljon G, Van Hees J, Van Den Abbeele J. Paternal Transmission of a Secondary Symbiont during Mating in the Viviparous Tsetse Fly. Mol Biol Evol. 2015;32:1977-80. 
122. Akman L, Rio RV, Beard CB, Aksoy S. Genome size determination and coding capacity of Sodalis glossinidius, an enteric symbiont of tsetse flies, as revealed by hybridization to Escherichia coli gene arrays. J Bacteriol. 2001; 183:4517-25.

123. Rio RV, Lefevre C, Heddi A, Aksoy S. Comparative genomics of insectsymbiotic bacteria: influence of host environment on microbial genome composition. Appl Environ Microbiol. 2003;69:6825-32.

124. Koch H, Schmid-Hempel P. Gut microbiota instead of host genotype drive the specificity in the interaction of a natural host-parasite system. Ecol Lett. 2012;15:1095-103.

125. Capewell P, Cren-Travaillé $C$, Marchesi F, Johnston P, Clucas C, Benson RA, Gorman TA, Calvo-Alvarez E, Crouzols A, Jouvion G, Jamonneau V, Weir W, Stevenson ML, O'Neill K, Cooper A, Swar NK, Bucheton B, Ngoyi DM, Garside P, Rotureau B, MacLeod A. The skin is a significant but overlooked anatomical reservoir for vector-borne African trypanosomes. Elife. 2016;5.

126. Casas-Sánchez A, Acosta-Serrano Á. Skin deep. elife. 2016;5.

127. Berthier $\mathrm{D}$, Brenière $\mathrm{SF}$, Bras-Gonçalves $\mathrm{R}$, Lemesre JL, Jamonneau V, Solano P, Lejon V, Thévenon S, Bucheton B. Tolerance to Trypanosomatids: A Threat, or a Key for Disease Elimination? Trends Parasitol. 2016;32(2):157-68.

Ready to submit your research? Choose BMC and benefit from:

- fast, convenient online submission

- thorough peer review by experienced researchers in your field

- rapid publication on acceptance

- support for research data, including large and complex data types

- gold Open Access which fosters wider collaboration and increased citations

- maximum visibility for your research: over $100 \mathrm{M}$ website views per year

At $\mathrm{BMC}$, research is always in progress.

Learn more biomedcentral.com/submissions 